26

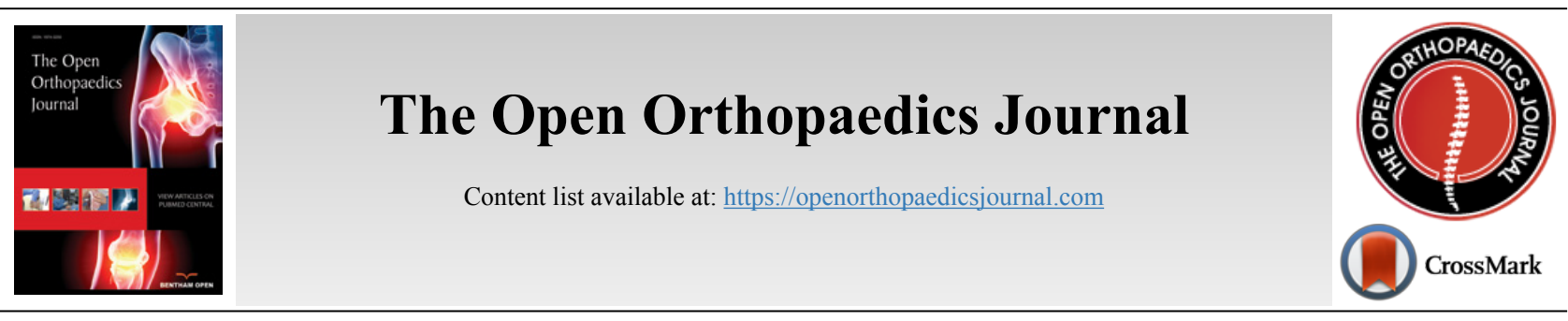

RESEARCH ARTICLE

\title{
A Retrospective Comparison of Early Postoperative Pain after the First $V s$ Second TKA in Scheduled Staged Bilateral TKA
}

\author{
Yoshinori Ishii ${ }^{1, *}$, Hideo Noguchi ${ }^{1}$, Junko Sato ${ }^{1}$, Hana Ishii ${ }^{2}$, Ryo Ishii ${ }^{3}$ and Shin-ichi Toyabe ${ }^{4}$ \\ ${ }^{1}$ Ishii Orthopedic \& Rehabilitation Clinic, 1089 Shimo-Oshi, Gyoda, Saitama, 361-002, Japan \\ ${ }^{2}$ Kanazawa Medical University, School of Plastic Surgery, 1-1 Daigaku Uchinada, Ishikawa 920-0253, Japan \\ ${ }^{3}$ Sado General Hospital, 161 Chikusa Sado, Niigata 952-1209, Japan \\ ${ }^{4}$ Niigata University Crisis Management Office, Niigata University Hospital, Niigata, Japan
}

\begin{abstract}
:
Background:

Acute postoperative pain control after Total Knee Arthroplasty (TKA) is important given that poorly controlled, persistent pain can delay rehabilitation. The purpose of this study was to compare pain intensity during the early postoperative period (following the first and second surgeries) in patients who underwent bilateral, scheduled, staged TKAs.

\section{Materials and Methods:}

We enrolled 32 patients (64 knees) in this study and evaluated the number of requests for analgesic agents during the first 3 days after TKA, time to walking, and the Wong-Baker FACES pain assessment score (WBS).

\section{Results:}

Comparing the postoperative period following the first and second TKA, there were no significant differences in WBS 24,48 , and $72 \mathrm{~h}$ postoperatively. The frequency of requests, and the total number of requests for analgesics did not differ when comparing the first and second TKA, at any time point. The total number of analgesic requests exhibited a moderately strong, positive correlation between the first and second TKA ( $\mathrm{p}<0.001, \mathrm{r}=0.623)$. Patients' WBS scores and requests for analgesics showed a moderately strong, positive correlation, but only at $24 \mathrm{~h}$ following the second TKA $(\mathrm{p}=0.002, \mathrm{r}=0.567)$. After both TKAs, patients required a median of 1 day to resume walking.

Conclusion:

Patients undergoing staged bilateral TKA experienced equivalent early postoperative pain when comparing their experience following their first and second TKAs. Therefore, regarding pain control following the second TKA, we recommend considering the analgesic administration schedule and requirements of the first TKA.
\end{abstract}

Keywords: Early postoperative pain, Staged bilateral total knee arthroplasty, Wong-Baker FACES pain assessment, Analgesics, Retrospective, Rehabilitation.

Article History

Received: August 29, 2019

Revised: December 25, 2019

Accepted: January 30, 2020

\section{INTRODUCTION}

The debate over the superiority of simultaneous versus staged Total Knee Arthroplasty (TKA) for patients with bilateral knee Osteoarthritis (OA) remains controversial regarding perioperative complications and economic factors [1 - 7]. Ritter et al. (1997) [5] reported that neither simultaneous nor staged bilateral TKA was clinically superior, and the deci-

\footnotetext{
* Address correspondence to this author at the Ishii Orthopedic and Rehabilitation Clinic, 1089 Shimo-Oshi, Gyoda, Saitama 361-0037, Japan, Tel: +81-11-81-485-55-3519; Fax: +81-11-81-485-55-3520,

E-mail: ishii@sakitama.or.jp
}

-sion to attempt simultaneous or staged bilateral TKA should be made on an individual basis by the patient and physician. The authors [4] recommended that preoperative health status be assessed carefully and that patients must be informed of both the risks and potential benefits of simultaneous bilateral TKA.

Regardless of the chosen procedure, pain control is important during the early postoperative period. Poorly controlled acute postoperative pain may lead to the development of chronic persistent pain [8,9]. Additionally, several studies [10 - 12] have reported that pain control differed between the first and second TKA. However, these 
studies evaluated pain mainly using subjective measures such as a simple Visual Analog Scale (VAS) $(0=$ no pain, $100=$ worst pain) [13].

In this study, we objectively assessed pain using the number of analgesic agent requests issued by each patient during the first 3 days after surgery and the time needed to resume walking because we considered that more severe pain delays the time to resume walking These assessments can be used with conventional qualitative scales such as the WongBaker FACES pain assessment scale (WBS) (0; No pain, 1; hurts a little bit, 2; hurts a little more, 3 ; hurts even more, 4; hurts a whole lot, 5 ; hurts the worst) [13]. The purpose of this study was to subjectively and objectively compare pain intensity during the early postoperative periods following the first and second TKA surgeries. The results may inform pain management strategies for patients who undergo staged, bilateral TKA.

\section{MATERIALS AND METHODS}

Informed consent was obtained from all patients. Approval for this study was granted by the Research Board of the Healthcare Corporation Ashinokai, Gyoda, Saitama, Japan (ID number: 2018-12). Potential participants were patients with OA who underwent bilateral, scheduled, staged TKAs with either a posterior cruciate ligament (PCL)-retaining (PCLR) design or a PCL-Substituting (PCLS) design between July 2009 and October 2018. All surgeries were performed using the LCS $^{\circledR}$ Total Knee System (DePuy, Warsaw, IN, USA) under general anesthesia.

Each patient individually selected the side for their first TKA. The timing of the second TKA was also determined by the patient and depended on his or her perceived ability to tolerate postoperative pain and activity limitations. The order of implant use was quasi-randomized; patients with even medical record numbers received a PCLR implant, and patients with odd medical record numbers received a PCLS implant during the first TKA. The second TKAs were performed using the implant design not selected for the first TKA. Unexpectedly, the manufacturer discontinued the PCL-R design at the end of January 2013; therefore, all subsequent TKAs were the PCLS design.

\subsection{Surgical Procedure and Postoperative Rehabilitation}

A single surgeon (YI) performed all procedures using a standardized technique, as described previously [16]. The femoral components were fixed without cement; however, cement was used for tibial component fixation in all patients. No patellae were resurfaced. Proper intraoperative anteroposterior and abduction/adduction stability were confirmed manually, although these were not quantified intraoperatively. All patients were able to achieve full extension, and at least $90^{\circ}$ of flexion, intraoperatively, in the supine position. No analgesics (intraoperatively) or peripheral nerve blocks (postoperatively) were used for pain control.

Postoperatively, all patients received a bulky compression dressing and intra-articular drains that were usually removed at the first dressing change. Full weight-bearing was allowed, as tolerated, using a cane, on postoperative day 1 under the supervision of a therapist, and exercises were allowed. Passive Range Of Motion (ROM) exercises were performed every day beginning 1 week after surgery. Patients received at least $2 \mathrm{~h}$ of daily physical therapy consisting of isometric exercises, passive ROM, active-assisted ROM, quadriceps and hamstring strengthening, gait training, and stair ascension and descent. All patients received perioperative prophylactic antibiotics and analgesics for pain. No patient received anti-thrombotics, although all patients received a venous foot pump device for approximately $2 \mathrm{~h}$ postoperatively.

\subsection{Outcomes Evaluation}

We used the WBS to rate postoperative pain severity because it correlates well with VAS scores [13]. We compared WBS scores following the first and second TKA at 24, 48, and $72 \mathrm{~h}$. These time points were selected according to previous studies indicating that $44 \%-57 \%$ of patients are awakened by pain during the first 3 postoperative days $[17,18]$. We asked patients to determine their WBS scores according to the most intense pain felt at each time point, regardless of the position of the knee. A combination of diclofenac sodium $(50 \mathrm{mg}$; suppository), ketoprofen (15 mg; intramuscular injection), and pentazocine (15 mg; intramuscular injection) was used for postoperative analgesia, according to each patient's request. Diclofenac sodium and ketoprofen are nonsteroidal antiinflammatory drugs that inhibit prostaglandin production at nociceptors. Pentazocine is a nonnarcotic analgesic that suppresses central nervous system conduction. We compared the frequencies with which each patient requested these analgesic agents, and the time before the resumption of walking during the postoperative periods (after the first and second TKAs) to objectively evaluate post-TKA pain. We also generated correlation coefficients for the number of analgesic requests and WBS scores at each time point following the first and second TKA.

\subsection{Statistical Analyses}

Normality assumptions were rejected by Q-Q plot, the Kolmogorov-Smirnov test, and the Shapiro-Wilk test. We used Wilcoxon's signed rank test for between-group comparisons of continuous variables and Spearman's rank correlation coefficient to test between-variable relationships. Correlative strengths were defined as follows: strong $=0.70-1.0$, moderate $=0.40-0.69$, or weak $=0.20-0.39$. The sample size to detect a difference at alpha (two-sided $=0.05$, power $=80 \%$ ) with an effect size of 0.3 was estimated to be 42 per group for Wilcoxon's signed rank test. The sample size to detect a significant correlation between two groups at alpha (two-sided $=0.05$, power $=80 \%$ ) with an effect size of 0.5 was estimated to be 34 per group for Spearman's rank correlation coefficient. Because the sample size of 32 per group was too small to detect statistical significance, we performed a post-hoc power analysis. This revealed a statistical power of $21 \%$ for Wilcoxon's signed rank test and $48 \%$ for Spearman's rank correlation coefficient. IBM SPSS Statistics ver. 23 (IBM Japan, Tokyo, Japan) was used to perform all statistical analyses. All values were expressed as medians [25th percentile, 75 th percentile], and for all tests, $p<0.05$ was considered statistically significant. 


\section{RESULTS}

Thirty-two patients (64 knees) who underwent bilateral, scheduled, staged TKAs with a PCLR design and a PCLS design, between July 2009 and October 2018, were enrolled in this study. The median interval between surgeries was 14 months (range: $6-77$ months). The median patient age at the time of the first surgery was 72 years (range: $63-83$ years). Preoperatively, all patients had been diagnosed with OA. Table 1 presents the patients' preoperative demographics. There were no adverse events induced by the analgesics used in this series. There were also no differences in WBS scores at postoperative 24,48 , and $72 \mathrm{~h}$ between the patients' first and second TKAs (Table 2).

Patients requested analgesics 102 times during the first 24 h, 43 times during the second $24 \mathrm{~h}$, and 63 times during the third $24 \mathrm{~h}$ following the first TKA. After the second TKA, total analgesic requests were 111 at $24 \mathrm{~h}, 43$ at $48 \mathrm{~h}$, and 63 at $72 \mathrm{~h}$ (Table 3). The observed differences between the total number of requests (both overall and at each time point) following the first and second TKA were non-significant. A comparison of the number of requests at each time point between the first and second TKA revealed a moderately strong, positive correlation $(\mathrm{p}<0.001, r=0.623)$ (Table 3). However, there were no significant correlations between WBS and the number of requests for analgesics, at each time point, following the first and second TKA, with the exception of the $24 \mathrm{~h}$ time point following the second TKA ( $p=0.002, r=0.567)$ (Table 4). Finally, patients required a median value of 1 day post-TKA to resume walking following both the first $[1,1]$ and second $[1,2]$ TKA, indicating no significant difference.

Table 1. Patients' characteristics and a general comparison of the first and second staged Total Knee Arthroplasty (TKA) procedures.

\begin{tabular}{|c|c|c|}
\hline Parameter & $1^{\text {st }}$ TKA $(n=32)$ & $2^{\text {nd }}$ TKA $(n=32)$ \\
\hline Age (yr) & $72[69,76]$ & $74[71,78]$ \\
\hline Sex (male/female) & $4 / 28$ & $4 / 28$ \\
\hline Weight $(\mathrm{kg})$ & $61[53,72]$ & $66[52,71]$ \\
\hline Height $(\mathrm{cm})$ & $150[146,155]$ & $149[145,156]$ \\
\hline BMI $\left(\mathrm{kg} / \mathrm{m}^{2}\right)$ & $27[23,31]$ & $27[24,31]$ \\
\hline ASA physical status score (I/II) & $0 / 32$ & $0 / 32$ \\
\hline Interval between $1^{\text {st }}$ and $2^{\text {nd }}$ TKA & \multicolumn{2}{|c|}{$14[11,23]$ (range; 6-77) } \\
\hline Preoperative WBS & $3[2,3]$ & $2[1,2]$ \\
\hline Operation time (min.) & $58[52,60]$ & $60[55,66]$ \\
\hline Tourniquet time (min) & $59[53,64]$ & $61[56,66]$ \\
\hline Anesthesia time (min) & $90[82,97]$ & $98[90,116]$ \\
\hline HSS Score & $43[34,49]$ & $48[36,54]$ \\
\hline $\mathrm{K} / \mathrm{L}$ Grade (III/IV) & $3 / 29$ & $4 / 28$ \\
\hline Preoperative WBS & $3[2,3]$ & $2[1,2]$ \\
\hline Design (PCLR/PCLS) & $7 / 25$ & $8 / 24$ \\
\hline
\end{tabular}

Values are expressed as $\mathrm{n}$ or median $\left[25^{\text {th }}\right.$ percentile, $75^{\text {th }}$ percentile]. BMI: body mass index, HSS score: Hospital for Special Surgery Score [14], K/L Grade: Kellgren-Lawrence grade [15], WBS: Wong-Baker FACES pain assessment scale [13], PCLR: posterior cruciate ligament-retaining meniscal-bearing type, PCLS: posterior cruciate ligament-sacrificing rotating platform type

Table 2. Comparison of the number of days prior to resumption of walking and the correlations in WBS scores at each time point between the first and second staged Total Knee Arthroplasty (TKA) procedures.

\begin{tabular}{|c|c|c|c|c|}
\hline Postoperative time & Time to walking (Days) & WBS 24h & WBS 48h & WBS 72h \\
\hline $1^{\text {st }}$ TKA & $1[1,1]$ (range 1 to 2$)$ & $3[3,4]$ & $3[3,4]$ & $3[2,3]$ \\
\hline $2^{\text {nd }}$ TKA & $1[1,2]($ range 1 to 3$)$ & $3[3,4]$ & $3[3,4]$ & $3[2,4]$ \\
\hline p values & 0.314 & 0.985 & 0.562 & 0.184 \\
\hline
\end{tabular}

Values are expressed as $n$ or median [25 percentile, 75 percentile].

WBS: Wong-Baker FACES pain assessment scale [13]

Table 3. Median and total frequencies of patients' requests for analgesics $24 \mathrm{~h}, 48 \mathrm{~h}$, and $72 \mathrm{~h}$ postoperatively $(\mathrm{n}=32)$.

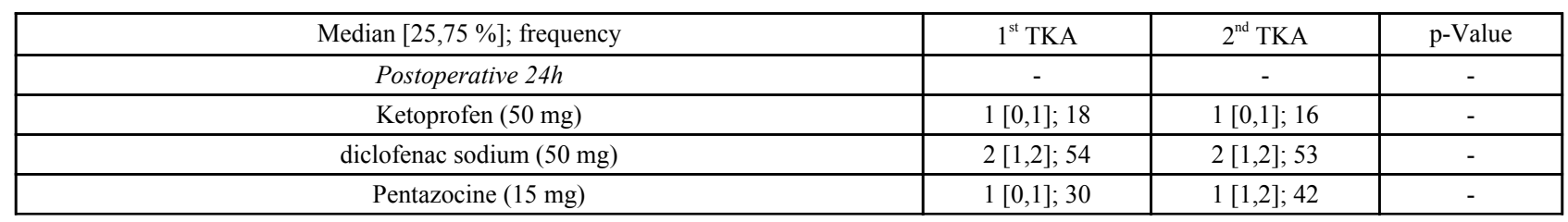


(Table 3) cont.....

\begin{tabular}{|c|c|c|c|}
\hline Total Frequency & 102 & 111 & 0.086 \\
\hline Postoperative $48 \mathrm{~h}$ & - & - & - \\
\hline Ketoprofen $(50 \mathrm{mg})$ & $0[0,0] ; 6$ & $0[0,0] ; 5$ & - \\
\hline diclofenac sodium $(50 \mathrm{mg})$ & $1[1,1] ; 31$ & $1[1,1] ; 32$ & - \\
\hline Pentazocine $(15 \mathrm{mg})$ & $0[0,0] ; 6$ & $0[0,0] ; 6$ & - \\
\hline Total Frequency & 43 & 43 & 0.921 \\
\hline Postoperative $72 \mathrm{~h}$ & - & - & - \\
\hline Ketoprofen $(50 \mathrm{mg})$ & $0[0,0] ; 0$ & $0[0,0] ; 2$ & - \\
\hline diclofenac sodium $(50 \mathrm{mg})$ & $2[2,2] ; 63$ & $2[2,2] ; 61$ & - \\
\hline Pentazocine $(15 \mathrm{mg})$ & $0[0,0] ; 0$ & $0[0,0] ; 0$ & - \\
\hline Total Frequency & 63 & 63 & 0.999 \\
\hline Accumulated Total frequency $(24 h+48 h+72 h)$ & 208 & 217 & $\begin{array}{c}0.323 \\
* p<0.001, \\
r=0.623\end{array}$ \\
\hline
\end{tabular}

TKA: total knee arthroplasty

$* \mathrm{p}<0.05$ was considered statistically significant.

Table 4. Correlation between WBS score and the frequency of analgesic requests at each time point between the first and second total knee arthroplasty (TKA) procedure.

\begin{tabular}{|c|c|c|c|}
\hline Postoperative time & $24 \mathrm{~h}$ & $48 \mathrm{~h}$ & $72 \mathrm{~h}$ \\
\hline $1^{\text {st }}$ TKA & 0.782 & 0.083 & 0.389 \\
\hline $2^{\text {nd }}$ TKA & $\mathrm{p}=0.002^{*}, \mathrm{r}=0.567$ & 0.329 & 0.973 \\
\hline
\end{tabular}

Values are expressed as $\mathrm{n}$ or median [25 percentile, 75 percentile]. WBS: Wong-Baker FACES pain assessment scale [13], ${ }^{*} \mathrm{p}<0.05$ was considered statistically significant.

\section{DISCUSSION}

There was no significant difference in the frequency of analgesic requests over the first 3 postoperative days following TKA. There was also no obvious correlation between the frequency with which analgesics were requested and WBS. Time to resumption of walking also did not vary significantly between the first and second TKA. However, patients' requests for analgesics exhibited a moderately strong, positive correlation between the first and second TKA. Therefore, early postoperative pain might be affected by differences in individual sensitivities, regardless of whether the TKA was the patient's first or second such procedure.

Various reports $[8,9,19]$ have indicated that poorly controlled acute postoperative pain can prolong pain $[8,9]$ and potentially delay postoperative rehabilitation [19]; thus, pain control is important. Other reports [10 - 12] compared acute pain intensity during the early postoperative period between the first and second TKA in patients who underwent staged bilateral TKA surgeries. The researchers concluded that patients required more analgesics after their second, compared with their first, TKA. The current comparative study provides additional information pertaining to pain control after a second TKA, adding to information provided in previous studies [10 - 12].

Kim et al. [10] compared early postoperative pain after staged bilateral TKA surgeries with a 1-week interval. The authors concluded that repeated surgery may induce hyperalgesia via central sensitization and postulated that this was likely the main reason patients required additional analgesics after their second TKA. Likewise, Sun et al. [12] reported that patients used more analgesics after their second
TKA, until $48 \mathrm{~h}$ postoperatively. The authors recommended performing a second TKA no fewer than 6 months after the first TKA to reduce or eliminate the hyperalgesic phenomenon induced by the first procedure. Other studies have also suggested that hyperalgesia secondary to central sensitization might affect the severity of postoperative pain. Central sensitization is reportedly induced by several factors, namely, harmful pain stimuli emanating from tissues around the operated joints $[20,21]$, persistent pain after the initial surgery $[22,23]$, and long-term pain stimuli secondary to severe OA [24]. However, our results differed from earlier findings [10 - 12]. Specifically, we found no difference in WBS scores between the first and second TKA, and no significant differences in the frequency with which analgesics were requested, or the time before patients resumed walking.

There are two potential explanations for our findings. The interval between TKA procedures ranged from 6-52 months (median: 14 months). This interval might have been long enough to diminish the influence of central sensitization evoked by the first TKA, in keeping with the suggestion made by Kim et al. [10]. This is consistent with findings reported by Sun et al. [12], who concluded that the second TKA should occur at least 6 months after the first TKA to mitigate the effects of central sensitization. The second, previouslyreported, factor is a psychological one [25, 26]. Pre- [25] and postoperative [26] anxiety can worsen pain after surgery. In this study, preoperative education did not vary between the first and second TKAs. However, it is reasonable to assume that patients might be less anxious about undergoing a second TKA, compared with their first procedure. The postoperative recovery process, including patients' familiarity with post- 
operative pain, may mitigate the fear and anxiety that would otherwise have accompanied a second procedure. Jiang et al. [27] reported findings that agreed with this hypothesis, stating that VAS anxiety scores [28] were lower in patients undergoing a second (second eye) cataract surgery. We believe that no matter what type of surgery, reduced presurgical anxiety likely exerts a positive effect on early postoperative pain, even assuming that some degree of hyperalgesia resulted from the first surgery [10 - 12]. Future studies quantitatively evaluating pre- and postoperative anxiety during staged bilateral TKA surgeries using validated assessment tools with minimal bias are needed and may help clarify the relationship between perioperative anxiety and the intensity of early postoperative pain.

Our results showed that patients who tended to frequently request pain medication after their first TKA also tended to issue frequent requests following their second TKA. Given that there were no correlations between WBS scores and requests for analgesics at each time point, for both the first and second TKA procedures, we cannot conclude that patients who perceived stronger pain requested additional medication. Additionally, our results might have been influenced by bias related to individual pain threshold differences; thus, postoperative pain management following a second TKA should be determined on a case-by-case basis. Our results suggest that practices such as increasing the dose of analgesics, or adding other analgesic modalities during the second postoperative period might not always be necessary. However, physicians should consider each patient's condition following the first TKA, when planning for a second procedure.

There were several limitations to this study. First and most important, this was a retrospective medical record and database review, which has inherent limitations. Secondly, the sample size was relatively small, although a power analysis confirmed that the number of participants was sufficient to detect differences. In addition, studies that make within-patient, rather than between-patient comparisons may be advantageous because the within-patient design controls for confounding variables and thus, fewer patients are required to obtain statistically reliable results [29]. Thirdly, WBS scores were not obtained at maximum flexion as in previous studies $[10,12]$ because of patients' limited ability to mobilize during the immediate postoperative period. However, instead of this measurement, we believe that the time until the resumption of walking was an objective measure of pain intensity to accomplish our purpose in this study. Fourthly, we compared requests for analgesics, not the actual amount of medication each patient was administered. The analgesics (nonsteroidal anti-inflammatory drugs and nonnarcotic analgesics) used in this series have different mechanisms of action and are associated with different patient medication sensitivities. Consequently, the order of use and personal preference could have affected our outcomes. Thus, we decided to compare only the frequency with which medications were requested. Finally, we did not use multimodal analgesics such as peripheral nerve blocks or periarticular infiltration analgesia, which are commonly used for postoperative pain control following current TKA surgeries [17, 30, 31]. However, the purpose of this study was to compare early postoperative pain intensity between the first and second TKA procedures using conventional analgesics. In addition, multimodal analgesic techniques are associated with certain limitations such as an increased risk of falls, delayed rehabilitation after nerve blocks [31], and disparities in the results related to infiltration techniques in periarticular infiltration analgesia [30].

Despite these limitations, a major strength of this study was our use of objective approaches to measure postoperative pain intensity after staged TKA, in addition to WBS scores. Furthermore, surgeon-related bias is not a consideration because all of the surgeries were completed by a single experienced surgeon who performed $>600$ TKA procedures using the current designs, and who used the same procedure and similar forms of instrumentation for all patients. Finally, the results of this study may be particularly useful for preemptive planning of perioperative pain control for the second knee replacement in patients who must undergo scheduled staged TKA surgery because of their preoperative health status.

\section{CONCLUSION}

In conclusion, patients with bilateral OA requested analgesics with a similar frequency, regardless of whether the TKA was their first or second. Additionally, the frequency with which patients requested analgesics did not vary significantly during the immediate postoperative period when we compared the first and second TKA procedures. The time needed prior to the resumption of walking also remained the same, when we compared the first and second TKAs. Therefore, we recommend that pain control following a second TKA be modeled after each patient's individualized usage patterns, as demonstrated following their earlier (first) TKA. Future largescale research studies are needed to both affirm, and build upon, our conclusions.

\section{ETHICS APPROVAL AND CONSENT TO PART- ICIPATE}

The study was approved by the Research Board of the Healthcare Corporation Ashinokai, Gyoda, Saitama, Japan (IDnumber: 2018-12).

\section{HUMAN AND ANIMAL RIGHTS}

No animals were used in this research. All human research procedures were followed in accordance with the ethical standards of the committee responsible for human experimentation (institutional and national), and with the Helsinki Declaration of 1975, as revised in 2013.

\section{CONSENT FOR PUBLICATION}

Informed consent was obtained from all patients. 


\section{AVAILABILITY OF DATA AND MATERIALS}

The data that support the findings of this study are available from the corresponding author, [Y.I.], upon reasonable request.

\section{FUNDING}

None.

\section{CONFLICT OF INTEREST}

Each author certifies that he or she has no commercial associations (e.g., consultancies, stock ownership, equity interest, patent/licensing arrangements) that might pose a conflict of interest in connection with the submitted article.

\section{ACKNOWLEDGEMENTS}

We thank Megumi Fukuda, RN, and Takeshi Yamamoto, $\mathrm{RN}$ for their contributions in gathering the data from Ishii Orthopedic \& Rehabilitation Clinic. We thank Jane Charbonneau, DVM, from Edanz Group (www.edanzediting.com/ac) for editing a draft of this manuscript.

\section{REFERENCES}

[1] Ersozlu S, Akkaya T, Ozgur AF, Sahin O, Senturk I, Tandogan R. Bilateral staged total knee arthroplasty in obese patients. Arch Orthop Trauma Surg 2008; 128(2): 143-8 [http://dx.doi.org/10.1007/s00402-007-0356-1] [PMID: 17694313]

[2] Fabi DW, Mohan V, Goldstein WM, Dunn JH, Murphy BP. Unilateral vs bilateral total knee arthroplasty risk factors increasing morbidity. J Arthroplasty 2011; 26(5): 668-73. [http://dx.doi.org/10.1016/j.arth.2010.07.011] [PMID: 20875943]

[3] Hutchinson JR, Parish EN, Cross MJ. A comparison of bilateral uncemented total knee arthroplasty: simultaneous or staged? J Bone Joint Surg Br 2006; 88(1): 40-3.

[http://dx.doi.org/10.1302/0301-620X.88B1.16454] [PMID: 16365118]

[4] Ritter MA, Harty LD, Davis KE, Meding JB, Berend M. Simultaneous bilateral, staged bilateral, and unilateral total knee arthroplasty. A survival analysis. J Bone Joint Surg Am 2003; 85(8): 1532-7. [http://dx.doi.org/10.2106/00004623-200308000-00015] [PMID: 12925634]

[5] Ritter M, Mamlin LA, Melfi CA, Katz BP, Freund DA, Arthur DS. Outcome implications for the timing of bilateral total knee arthroplasties. Clin Orthop Relat Res 1997; (345): 99-105. [http://dx.doi.org/10.1097/00003086-199712000-00014] [PMID: 9418626]

[6] Stefánsdóttir A, Lidgren L, Robertsson O. Higher early mortality with simultaneous rather than staged bilateral TKAs: Results from the swedish knee arthroplasty register. Clin Orthop Relat Res 2008; 466(12): 3066-70

[http://dx.doi.org/10.1007/s11999-008-0404-3] [PMID: 18670832]

[7] Yoon HS, Han CD, Yang IH. Comparison of simultaneous bilateral and staged bilateral total knee arthroplasty in terms of perioperative complications. J Arthroplasty 2010; 25(2): 179-85.

[http://dx.doi.org/10.1016/j.arth.2008.11.103] [PMID: 19195827]

[8] Fuzier R, Serres I, Bourrel R, Palmaro A, Montastruc JL, LapeyreMestre M. Analgesic drug consumption increases after knee arthroplasty: A pharmacoepidemiological study investigating postoperative pain. Pain 2014; 155(7): 1339-45. [http://dx.doi.org/10.1016/j.pain.2014.04.010] [PMID: 24727347]

[9] Voscopoulos C, Lema M. When does acute pain become chronic? Br J Anaesth 2010; 105(Suppl. 1): i69-85.

[http://dx.doi.org/10.1093/bja/aeq323] [PMID: 21148657]

[10] Kim MH, Nahm FS, Kim TK, Chang MJ, Do SH. Comparison of postoperative pain in the first and second knee in staged bilateral total knee arthroplasty: clinical evidence of enhanced pain sensitivity after surgical injury. Pain 2014; 155(1): 22-7.

[http://dx.doi.org/10.1016/j.pain.2013.08.027] [PMID: 23994101]
[11] Shin HJ, Kim EY, Na HS, Kim TK, Kim MH, Do SH. Magnesium sulphate attenuates acute postoperative pain and increased pain intensity after surgical injury in staged bilateral total knee arthroplasty: A randomized, double-blinded, placebo-controlled trial. Br J Anaesth 2016; 117(4): 497-503.

[http://dx.doi.org/10.1093/bja/aew227] [PMID: 28077538]

[12] Sun J, Li L, Yuan S, Zhou Y. Analysis of early postoperative pain in the first and second knee in staged bilateral total knee arthroplasty: A retrospective controlled study. PLoS One 2015; 10(6)e0129973 [http://dx.doi.org/10.1371/journal.pone.0129973] [PMID: 26068371]

[13] Garra G, Singer AJ, Taira BR, et al. Validation of the wong-baker faces pain rating scale in pediatric emergency department patients. Acad Emerg Med 2010; 17(1): 50-4.

[http://dx.doi.org/10.1111/j.1553-2712.2009.00620.x]

[PMID: 20003121]

[14] Alicea J. Scoring systems and their validation for the arthritic knee.Surgery of the knee. $3^{\text {rd }}$ ed. New York: Churchill Livingstone 2001; 2: pp. 1507-15.

[15] Kellgren JH, Lawrence JS. Radiological assessment of osteo-arthrosis Ann Rheum Dis 1957; 16(4): 494-502. [http://dx.doi.org/10.1136/ard.16.4.494] [PMID: 13498604]

[16] Ishii Y, Noguchi H, Takeda M, Sato J, Ezawa N, Toyabe S. Changes in lower extremity 3-dimensional load-bearing axes before and after mobile-bearing total knee arthroplasty. J Arthroplasty 2012; 27(6): 1203-9.

[http://dx.doi.org/10.1016/j.arth.2011.12.013] [PMID: 22305436]

[17] Grosu I, Lavand'homme P, Thienpont E. Pain after knee arthroplasty: an unresolved issue. Knee Surg Sports Traumatol Arthrosc 2014; 22(8): 1744-58.

[http://dx.doi.org/10.1007/s00167-013-2750-2] [PMID: 24201900]

[18] Wylde V, Rooker J, Halliday L, Blom A, Halliday L, Blom A. Acute postoperative pain at rest after hip and knee arthroplasty: Severity, sensory qualities and impact on sleep. Orthop Traumatol Surg Res 2011; 97(2): 139-44

[http://dx.doi.org/10.1016/j.otsr.2010.12.003] [PMID: 21388906]

[19] Capdevila X, Barthelet Y, Biboulet P, Ryckwaert Y, Rubenovitch J, d'Athis F. Effects of perioperative analgesic technique on the surgical outcome and duration of rehabilitation after major knee surgery. Anesthesiology 1999; 91(1): 8-15.

[http://dx.doi.org/10.1097/00000542-199907000-00006] [PMID: 10422923]

[20] Woolf CJ. Central sensitization: implications for the diagnosis and treatment of pain. Pain 2011; 152(3)(Suppl.): S2-S15.

[http://dx.doi.org/10.1016/j.pain.2010.09.030] [PMID: 20961685]

[21] Woolf CJ, Salter MW. Neuronal plasticity: increasing the gain in pain. Science 2000; 288(5472): 1765-9.

[http://dx.doi.org/10.1126/science.288.5472.1765] [PMID: 10846153]

[22] Suzuki R, Dickenson A. Spinal and supraspinal contributions to central sensitization in peripheral neuropathy. Neurosignals 2005 ; 14(4): 175-81.

[http://dx.doi.org/10.1159/000087656] [PMID: 16215300]

[23] Wieseler-Frank J, Maier SF, Watkins LR. Glial activation and pathological pain. Neurochem Int 2004; 45(2-3): 389-95. [http://dx.doi.org/10.1016/j.neuint.2003.09.009] [PMID: 15145553]

[24] Arendt-Nielsen L, Nie H, Laursen MB, et al. Sensitization in patients with painful knee osteoarthritis. Pain 2010; 149(3): 573-81. [http://dx.doi.org/10.1016/j.pain.2010.04.003] [PMID: 20418016]

[25] Sjöling M, Nordahl G, Olofsson N, Asplund K. The impact of preoperative information on state anxiety, postoperative pain and satisfaction with pain management. Patient Educ Couns 2003; 51(2): 169-76.

[http://dx.doi.org/10.1016/S0738-3991(02)00191-X] [PMID: 14572947]

[26] Pinto PR, McIntyre T, Ferrero R, Almeida A, Araújo-Soares V. Risk factors for moderate and severe persistent pain in patients undergoing total knee and hip arthroplasty: A prospective predictive study. PLoS One 2013; 8(9)e 73917

[http://dx.doi.org/10.1371/journal.pone.0073917] [PMID: 24058502]

[27] Jiang L, Zhang K, He W, Zhu X, Zhou P, Lu Y. Perceived Pain during Cataract Surgery with Topical Anesthesia: A Comparison between First-Eye and Second-Eye Surgery. J Ophthalmol 2015; 2015383456 [http://dx.doi.org/10.1155/2015/383456] [PMID: 26064671]

[28] Millar K, Jelicic M, Bonke B, Asbury AJ. Assessment of preoperative anxiety: comparison of measures in patients awaiting surgery for breast cancer. Br J Anaesth 1995; 74(2): 180-3. [http://dx.doi.org/10.1093/bja/74.2.180] [PMID: 7696068]

[29] Pritchett JW. Patients prefer a bicruciate-retaining or the medial pivot 
total knee prosthesis. J Arthroplasty 2011; 26(2): 224-8.

[http://dx.doi.org/10.1016/j.arth.2010.02.012] [PMID: 20932707]

[30] Mont MA, Beaver WB, Dysart SH, Barrington JW, Del Gaizo DJ.

Local infiltration analgesia with liposomal bupivacaine improves pain scores and reduces opioid use after total knee arthroplasty: Results of a randomized controlled trial. J Arthroplasty 2018; 33(1): 90-6. [http://dx.doi.org/10.1016/j.arth.2017.07.024] [PMID: 28802777]

[31] Yu S, Dundon J, Solovyova O, Bosco J, Iorio R. Can multimodal pain management in tka eliminate patient-controlled analgesia and femoral nerve blocks? Clin Orthop Relat Res 2018; 476(1): 101-9.

[http://dx.doi.org/10.1007/s11999.0000000000000018]

[PMID:

\section{(C) 2020 Ishii et al.}

This is an open access article distributed under the terms of the Creative Commons Attribution 4.0 International Public License (CC-BY 4.0), a copy of which is available at: https://creativecommons.org/licenses/by/4.0/legalcode. This license permits unrestricted use, distribution, and reproduction in any medium, provided the original author and source are credited. 